\title{
SPH Simulation of Acoustic Waves: Effects of Frequency, Sound Pressure, and Particle Spacing
}

\author{
Y. O. Zhang, ${ }^{1,2}$ T. Zhang, ${ }^{1,3}$ H. Ouyang, ${ }^{4}$ and T. Y. Li ${ }^{1,3}$ \\ ${ }^{1}$ School of Naval Architecture and Ocean Engineering, Huazhong University of Science and Technology, Wuhan 430074, China \\ ${ }^{2}$ Department of Mechanical and Aerospace Engineering, University of California, San Diego, La Jolla, CA 92093, USA \\ ${ }^{3}$ Hubei Key Laboratory of Naval Architecture and Ocean Engineering Hydrodynamics, Huazhong University of Science and Technology, \\ Wuhan 430074, China \\ ${ }^{4}$ School of Engineering, University of Liverpool, The Quadrangle, L69 $3 G H$ Liverpool, UK
}

Correspondence should be addressed to T. Zhang; zhangt7666@mail.hust.edu.cn

Received 30 August 2014; Revised 15 November 2014; Accepted 15 November 2014

Academic Editor: Kim M. Liew

Copyright (C) 2015 Y. O. Zhang et al. This is an open access article distributed under the Creative Commons Attribution License, which permits unrestricted use, distribution, and reproduction in any medium, provided the original work is properly cited.

Acoustic problems consisting of multiphase systems or with deformable boundaries are difficult to describe using mesh-based methods, while the meshfree, Lagrangian smoothed particle hydrodynamics (SPH) method can handle such complicated problems. In this paper, after solving linearized acoustic equations with the standard SPH theory, the feasibility of the SPH method in simulating sound propagation in the time domain is validated. The effects of sound frequency, maximum sound pressure amplitude, and particle spacing on numerical error and time cost are then subsequently discussed based on the sound propagation simulation. The discussion based on a limited range of frequency and sound pressure demonstrates that the rising of sound frequency increases simulation error, and the increase is nonlinear, whereas the rising sound pressure has limited effects on the error. In addition, decreasing the particle spacing reduces the numerical error, while simultaneously increasing the CPU time. The trend of both changes is close to linear on a logarithmic scale.

\section{Introduction}

Some classic numerical methods such as the finite element method (FEM) $[1,2]$, the boundary element method (BEM) [3], and other modified or coupled methods [4-6] are widely used for acoustic simulations. However, these mesh-based methods are not ideal for solving acoustic problems consisting of a variety of media or with deformable boundaries.

Meshfree methods can handle such complicated problems. The method of fundamental solutions (MFS) [7], the multiple-scale reproducing kernel particle method (RKPM) [8], the element-free Galerkin method (EFGM) [9], and other meshfree methods $[10,11]$ have been applied to these acoustic problems.

The smoothed particle hydrodynamics (SPH) method, as a meshfree, Lagrangian method, was first independently pioneered by Lucy [12] and Gingold and Monaghan [13] to solve astrophysical problems in 1977. In addition, the SPH method has been used in many different fields [14-16]. It not only has most advantages of a meshfree method, but also is suitable for solving problems with material separation or large ranges of density as illustrated in recent reviews by Li and Liu [17], Springel [18], M. B. Liu and G. R. Liu [19], and Monaghan [20] due to its Lagrangian property. Introducing the SPH method to acoustic computation also brings its advantages to some fields like bubble acoustic, combustion noise, sound propagation in multiphase flows, and so on.

With the advance of the SPH method in acoustic simulation, some research literatures [21,22] discussed solving fluid dynamic equations to simulate sound waves. In addition, we published a conference paper [23] that used the SPH method to solve linearized acoustic equations for modeling sound propagation and interference. Numerical results showed that the SPH method was capable of accurately modeling sound propagation, but the effects of frequency and sound pressure on the SPH simulation need further discussion. Therefore, the 
present paper focuses on discussing the effect of frequency and sound pressure of the acoustic waves on the numerical error caused by the SPH simulation.

The present paper is organized as follows. In Section 2, the standard SPH theory is used to solve the linearized acoustic equations. In Section 3, a one-dimensional sound propagation model is built. In Section 4, a numerical experiment is given based on standard SPH algorithms, and the effect of frequency, sound pressure, and particle spacing on the simulation is analyzed with considering the changes of particle spacing and Courant number. Section 5 summarizes the results of this work.

\section{SPH Formulations of Sound Waves}

2.1. Basic Formulations of SPH. As a meshfree, Lagrangian particle method, the SPH method is an important method widely used in recent years. Formulations in the SPH theory are represented in a particle approximation form. The properties of each particle are computed using an interpolation process over its neighboring particles [24]. In this way, the integral of a field function $f(\mathbf{r})$ can be represented as

$$
\langle f(\mathbf{r})\rangle=\int_{\Omega} f\left(\mathbf{r}^{\prime}\right) W\left(\mathbf{r}-\mathbf{r}^{\prime}, h\right) \mathrm{d} \mathbf{r}^{\prime},
$$

where $\langle\cdot\rangle$ is the kernel approximation operator, $f$ is a function of the vector $\mathbf{r}, \Omega$ is the volume of the integral, $W$ is the smoothing kernel, and $h$ is the smoothing length.

The particle approximation for the function $f(\mathbf{r})$ at particle $i$ within the support domain can be written as

$$
\left\langle f\left(\mathbf{r}_{i}\right)\right\rangle=\sum_{j=1}^{N} \frac{m_{j}}{\rho_{j}} f\left(\mathbf{r}_{j}\right) \cdot W_{i j},
$$

where $\mathbf{r}_{i}$ and $\mathbf{r}_{j}$ are the position of particles $i$ and $j, N$ is the number of particles in the computational domain, $m_{j}$ is the mass of particle $j, W_{i j}=W\left(r_{i j}, h\right)$, and $r_{i j}$ is the distance between particle $i$ and particle $j$.

Similarly, the gradient of function $f(\mathbf{r})$ at particle $i$ is obtained as

$$
\left\langle\nabla \cdot f\left(\mathbf{r}_{i}\right)\right\rangle=\sum_{j=1}^{N} \frac{m_{j}}{\rho_{j}} f\left(\mathbf{r}_{j}\right) \cdot \nabla_{i} W_{i j},
$$

where $\nabla_{i} W_{i j}=\left(\left(\mathbf{r}_{i}-\mathbf{r}_{j}\right) / r_{i j}\right)\left(\partial W_{i j} / \partial r_{i j}\right)$.

2.2. SPH Formulations of Sound Waves. In acoustic simulation, the governing equations for constructing SPH formulations are the laws of continuity, momentum, and state. The simplest and most common acoustical problem occurs under some assumptions. On one hand, the medium is lossless and at rest, so an energy equation is unnecessary; on the other hand, a small departure from quiet conditions occurs as follows:

$$
\begin{array}{cc}
\rho=\rho_{0}+\delta \rho, & |\delta \rho| \ll \rho_{0} \\
P=p_{0}+p, & |p| \ll \rho_{0} c_{0}^{2} \\
\mathbf{u}=0+\mathbf{u}, & |\mathbf{u}| \ll c_{0},
\end{array}
$$

where $\rho$ is the fluid density, $\rho_{0}$ is the quiescent density which does not vary in time and space, $\delta \rho$ is the change of density, $P$ is the instantaneous pressure at time $t$ of the fluid, $p_{0}$ is the quiescent pressure, $p$ is the sound pressure, $c_{0}$ is the speed of sound, and $\mathbf{u}$ is the flow velocity. $\delta \rho, p$, and $\mathbf{u}$ are taken to be small quantities of first order.

By discarding second-order terms in the acoustic equations, the linearized continuity, momentum, and state equations (for ideal air) governing sound waves are obtained as

$$
\begin{aligned}
\frac{\partial(\delta \rho)}{\partial t} & =-\rho \nabla \cdot \mathbf{u} \\
\frac{\partial \mathbf{u}}{\partial t} & =-\frac{1}{\rho} \nabla p \\
p & =c_{0}^{2} \delta \rho .
\end{aligned}
$$

Applying the SPH particle approximation (see (3)) to (7), the particle approximation equation of the continuity of acoustic waves is written as

$$
\frac{\partial\left(\delta \rho_{i}\right)}{\partial t}=\left(\rho_{0}+\delta \rho_{i}\right) \sum_{j=1}^{N} \frac{m_{j}}{\left(\rho_{0}+\delta \rho_{j}\right)} \mathbf{u}_{i j} \nabla_{i} W_{i j},
$$

where $\rho_{0}$ is the quiescent density which does not vary in time and space, $p_{0}$ is the quiescent pressure, and $\mathbf{u}_{i j}=\mathbf{u}_{i}-\mathbf{u}_{j}$.

The momentum equation in SPH method is obtained as

$$
\frac{\partial \mathbf{u}_{i}}{\partial t}=\sum_{j=1}^{N} m_{j}\left[\frac{p_{i}}{\left(\rho_{0}+\delta \rho_{i}\right)^{2}}+\frac{p_{j}}{\left(\rho_{0}+\delta \rho_{j}\right)^{2}}\right] \nabla_{i} W_{i j}
$$

Particle approximation of the equation of state is

$$
p_{i}=c_{0}^{2} \delta \rho_{i}
$$

The cubic spline function given by Monaghan and Lattanzio [25] is used as the smoothing kernel in this paper, which is written as

$$
W(r, h)=\alpha_{D} \begin{cases}1-\frac{3}{2} q^{2}+\frac{3}{4} q^{3} & 0 \leq q \leq 1 \\ \frac{1}{4}(2-q)^{3} & 1 \leq q \leq 2 \\ 0 & q \geq 2,\end{cases}
$$

where $\alpha_{D}$ is $1 / h$ in one-dimensional space, $q=r / h, r$ is the distance between two particles, and $h$ is the smoothing length which defines the influence area of smoothing function $W$.

The second-order leap-frog integration [26] is used in the present paper. All-pair search approach [24], as a direct and simple algorithm, is used to realize the neighbor particles searching in the acoustic wave simulation.

\section{Sound Propagation Model}

In order to evaluate the effect of sound pressure and frequency, a one-dimensional sound wave which propagates in a pipe with uniform cross section is used. The acoustic model is shown in Figure 1. The sound pressure at $t=0$ is plotted with 
TABLE 1: Model parameters and computational parameters for the numerical experiment.

\begin{tabular}{lcccc}
\hline \multirow{2}{*}{ Model parameters } & $p_{\text {Amp }}$ & $w$ & $c_{0}$ & $t$ \\
& $50 \mathrm{~Pa}$ & $50 \mathrm{rad} / \mathrm{s}$ & $340 \mathrm{~m} / \mathrm{s}$ & $0.1 \mathrm{~s}$ \\
\hline \multirow{2}{*}{ Computational parameters } & Particle spacing & Smoothing length & Courant number & Particle mass \\
& $0.04 \mathrm{~m}$ & $0.13 \mathrm{~m}$ & 0.05 & $0.04 \mathrm{~kg}$ \\
\hline
\end{tabular}

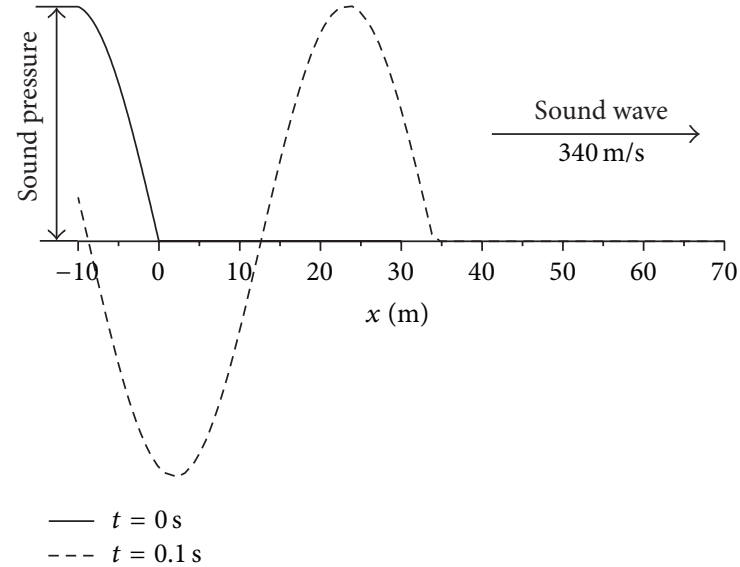

FIgURE 1: One-dimensional sound propagation model.

a solid line, while the sound pressure at $t=0.1 \mathrm{~s}$ is plotted with a dashed line.

Sound pressure of the source of sound wave in Figure 1 is

$$
p(t, x<0)=p_{\text {Amp }} \sin (w t-k x),
$$

where $t$ represents time (propagation starts when $t=0$ ), $x$ is the geometric position, $p_{\text {Amp }}$ is the maximum sound pressure of the acoustic wave, $w$ is the circular frequency of the acoustic wave, $k=w / c_{0}$ is the wave number, and the sound speed $c_{0}$ is $340 \mathrm{~m} / \mathrm{s}$.

The sound propagates from $x<0$ to $x \geq 0$, with the $\mathrm{SPH}$ computational region going from $-10 \mathrm{~m}$ to $70 \mathrm{~m}$. In the computation, the region of the sound source is from $-10 \mathrm{~m}$ to $0 \mathrm{~m}$, which is determined by the theoretical solutions, while the region of sound propagation is from $0 \mathrm{~m}$ to $70 \mathrm{~m}$, which is obtained by the SPH method. At the end of the sound propagation domain, the particles are set free in the computation. Since the SPH computational region is far longer than the sound propagation distance, any effects caused by ignoring this boundary will not propagate to the sound propagation domain and therefore can be neglected. The simulation results at the time $t=0.1 \mathrm{~s}$ are used to compare with the theoretical solutions. A similar numerical model is used in our recent conference paper [23].

In the evaluation of numerical results, the nondimensional simulation error of the sound pressure is defined by

$$
\varepsilon_{\text {pre }}=\frac{1}{N \cdot p_{\text {Amp }}} \sqrt{\sum_{j=1}^{N}\left(p_{j}^{*}-p\left(x_{j}\right)\right)^{2}},
$$

where $p_{j}^{*}$ is the simulation pressure of the particle $j$ and $p\left(x_{j}\right)$ is the theoretical pressure at the position of particle $j$.

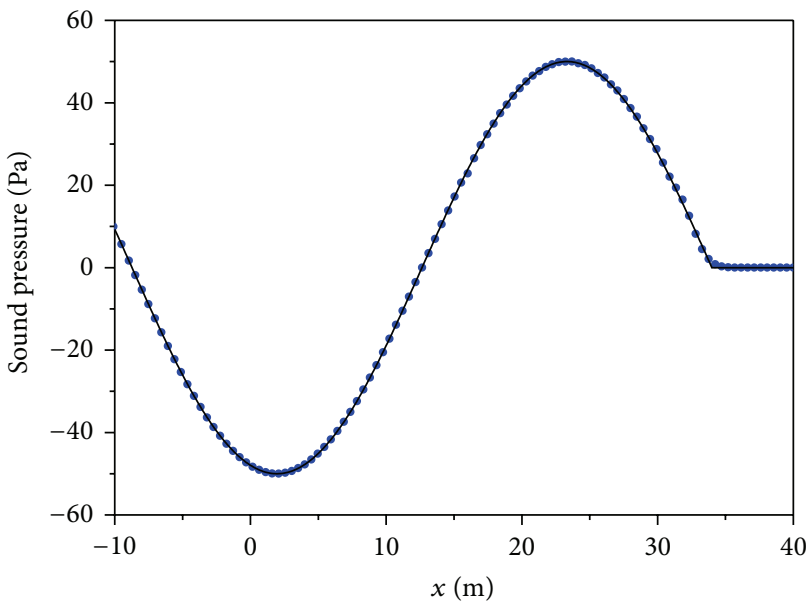

- $\mathrm{SPH}$

- Theoretical

FIGURE 2: Sound pressure comparison between SPH results and theoretical solutions. ( $p_{\text {Amp }}=50 \mathrm{~Pa}, w=50 \mathrm{rad} / \mathrm{s}$, and $t=0.1 \mathrm{~s}$ ).

\section{Results and Discussion}

4.1. Numerical Experiment. A sound propagation model is built to confirm agreement between SPH simulation results and the theoretical solution. The parameters used in the numerical experiment of the sound waves are listed in Table 1 . The physical time required for the modeling was 141.6 seconds.

A comparison between the SPH results and theoretical solutions of sound pressure at $t=0.1 \mathrm{~s}$ is shown in Figure 2 . The theoretical solution is plotted with a solid line while the SPH results are plotted with red points. In order to clearly identify the numerical results, the points are plotted at intervals of 12 .

As shown in Figure 2, the sound pressure of the sound wave changes along with the position, and a peak and a valley appear in the final wave at $t=0.1 \mathrm{~s}$. Comparing with the analytical data, it can be seen that the SPH method can accurately simulate the sound pressure of an acoustic wave as the values of sound pressure can be obtained precisely, and the simulation error is $1.6 \times 10^{-4}$. The trend of both the $\mathrm{SPH}$ results and the theoretical solutions also has a similar tendency. 


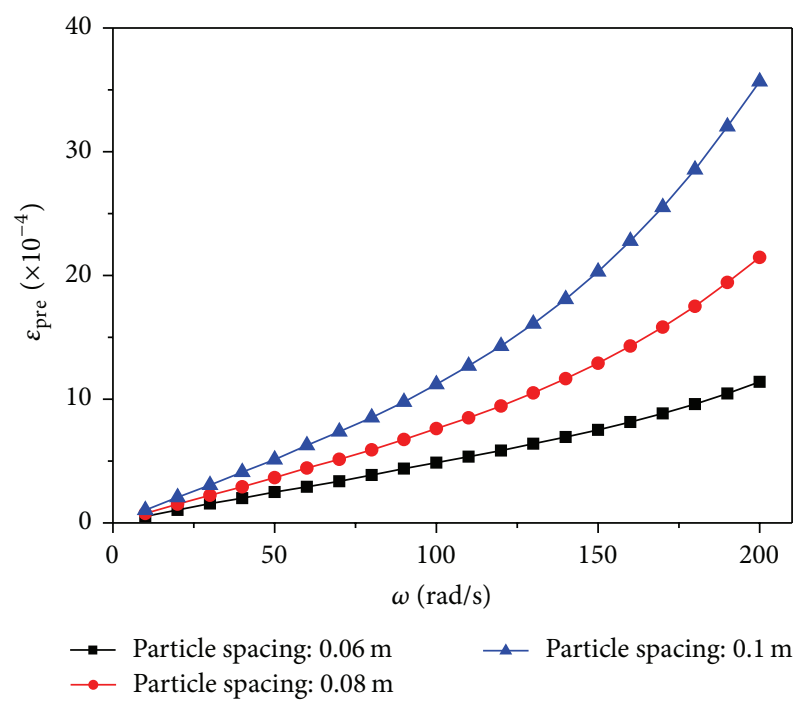

Figure 3: Sound pressure error versus circular frequency for different particle spacings.

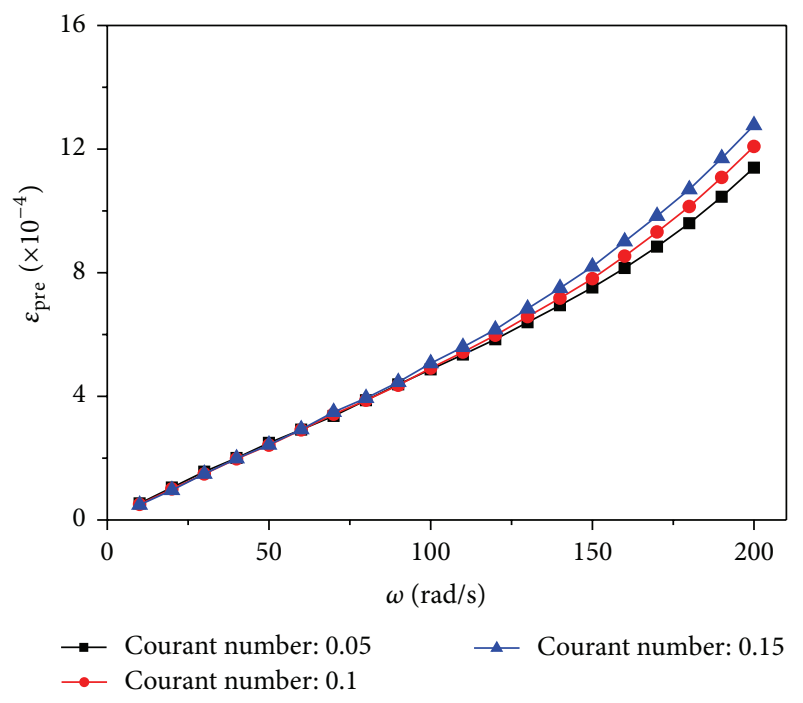

FIGURE 4: Sound pressure error versus circular frequency for different Courant numbers.

4.2. Effect of Frequency. Different sound propagation models with the circular frequency of sound changing from $10 \mathrm{rad} / \mathrm{s}$ to $200 \mathrm{rad} / \mathrm{s}$ are simulated, and the effect of circular frequency is discussed. In the discussion, different computational parameters, namely, the particle spacing and the Courant number, are also considered.

Figure 3 gives the numerical error for different computational cases with different $w$ and particle spacing. Figure 4 shows the sound pressure error for different cases with different values of circular frequency and Courant number. Each point in both figures stands for a computational case. The Courant number used in the computational cases in Figure 3 is 0.05 , and the particle spacing used in all cases in Figure 4 is $0.06 \mathrm{~m}$.

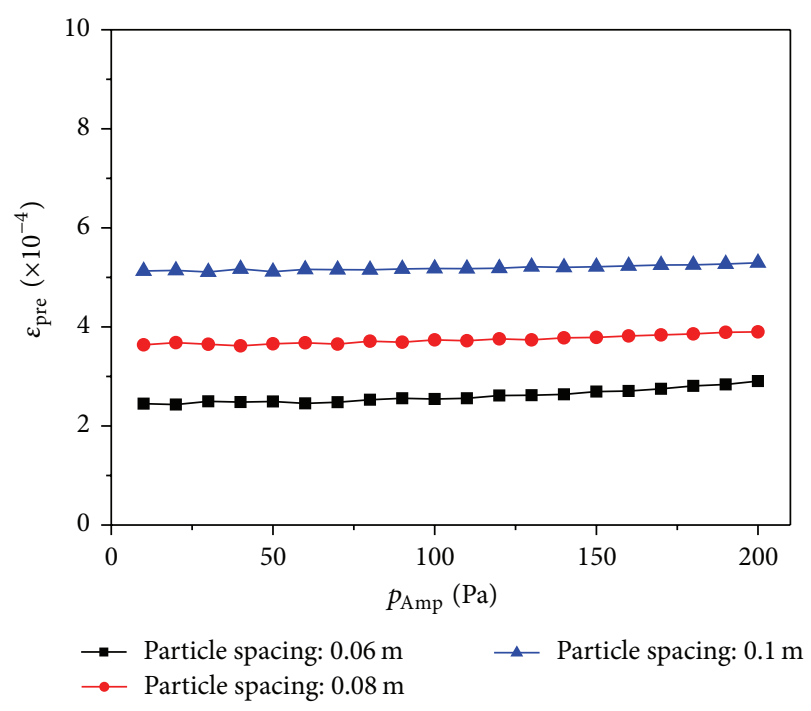

FIGURE 5: Sound pressure error versus maximum sound pressure amplitude for different particle spacings.

As can be seen from Figure $3, \varepsilon_{\text {pre }}$ increases along with the increase of $w$, and the increase of $\varepsilon_{\text {pre }}$ is nonlinear. Along with the increase of $w$, the increase of $\varepsilon_{\text {pre }}$ is larger. On the other hand, we can see from the line graph that $\varepsilon_{\text {pre }}$ is much larger than other positions when $w$ is $200 \mathrm{rad} / \mathrm{s}$. The numerical error grows faster when the particle spacing is larger.

As shown in Figure $4, \varepsilon_{\text {pre }}$ increases along with the increase of $w$. What is more, three lines are represent three different Courant numbers, namely, three different choices of time step when the particle spacing is the same. $\varepsilon_{\text {pre }}$ obtained from three different Courant numbers has almost the same value when $w$ is under $100 \mathrm{rad} / \mathrm{s}$. After that, some difference is shown among three lines when $w$ becomes larger, but the Courant number affects the effect of $w$ less in general.

4.3. Effect of Sound Pressure. In the present section, different sound propagation models with the maximum sound pressure amplitude changing from $10 \mathrm{~Pa}$ to $200 \mathrm{~Pa}$ are simulated, and the effects of sound pressure are discussed. At the same time, different computational parameters, namely, the particle spacing and the Courant number, are also used in the simulation. The $w$ of the sound in all computational cases in this section is $50 \mathrm{rad} / \mathrm{s}$.

Figure 5 illustrates the changes of sound pressure error when the numerical model uses different $p_{\text {Amp }}$ and particle spacing. Figure 6 describes the changes of error with different $p_{\text {Amp }}$ and different Courant numbers. Each point in both figures stands for a computational case. The Courant number used in all computational cases in Figure 5 is 0.05 , and the particle spacing used in all cases in Figure 6 is $0.06 \mathrm{~m}$.

It can be seen from the line graph that $\varepsilon_{\text {pre }}$ has limited change, which is a little increase, along with the increase of $p_{\text {Amp }}$. The trend of the line is approximately linear when $p_{\text {Amp }}$ changes from $10 \mathrm{~Pa}$ to $200 \mathrm{~Pa}$. The value of three lines in the figure is around $2.7 \times 10^{-4}, 3.7 \times 10^{-4}$, and $5.2 \times 10^{-4}$ corresponding to the particle spacing changes from $0.06 \mathrm{~m}$ 


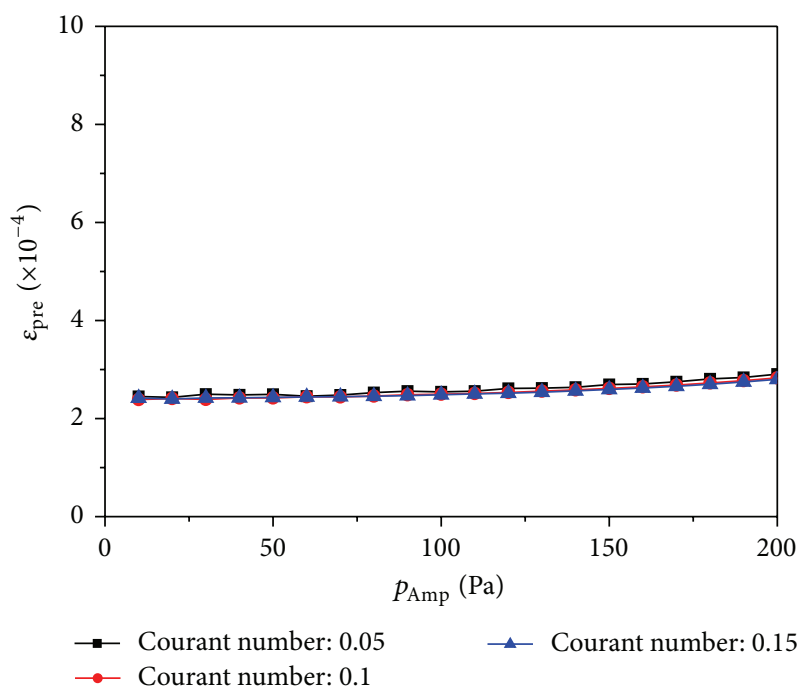

FIGURE 6: Sound pressure error versus maximum sound pressure amplitude for different Courant numbers.

to $0.1 \mathrm{~m}$. In other words, when the particle spacing increases, the error shows an upward trend.

Similarly, $\varepsilon_{\text {pre }}$ rises a little along with the increase of the amplitude of sound wave as shown in Figure 6. Specifically, $\varepsilon_{\text {pre }}$ stays at about $2.6 \times 10^{-4}$ when the amplitude of sound changes from $10 \mathrm{~Pa}$ to $200 \mathrm{~Pa}$. Although with different Courant number, the error of numerical results has similar values. The largest difference of $\varepsilon_{\text {pre }}$ among three lines is $2.0 \times$ $10^{-5}$, which happens when $p_{\text {Amp }}$ reaches $200 \mathrm{~Pa}$.

4.4. Effect of Particle Spacing. In addition to the accuracy, the convergence and the computational efficiency are also worth concern. Particle spacing, as an important parameter in the SPH simulation, affects these indexes a lot. In this section, the effects of the particle spacing are analyzed based on the model in Section 4.1 with the particle spacing $(\Delta x)$ changes from $0.03 \mathrm{~m}$ to $0.3 \mathrm{~m}$.

Different sound pressure errors corresponding to the SPH simulation results with different $\Delta x$ are shown in Figure 7. Three different types of points are used in the figure to represent Courant numbers of $0.05,0.10$, and 0.15 in the computation. In order to clearly show the trend, logarithmic scales are used for both axes in the figure.

As can be seen from Figure 7, there is an approximately linear relation between $\varepsilon_{\text {pre }}$ and $\Delta x$ with double logarithmic coordinates. As $\Delta x$ increases, $\varepsilon_{\text {pre }}$ also increases. This result indicates a convergence of the simulation, as a smaller $\Delta x$ leads to a more accurate result. Note that the errors generated with the three different Courant numbers all have similar values. A linear fitted curve obtained by using all $\varepsilon_{\text {pre }}$ results produces a slope of approximately 1.4 for the fitted line.

The required CPU cost in terms of time to perform the SPH simulation corresponding to different $\Delta x$ values is given in Figure 8. Three different point types were also used to represent different Courant numbers in the computation. The

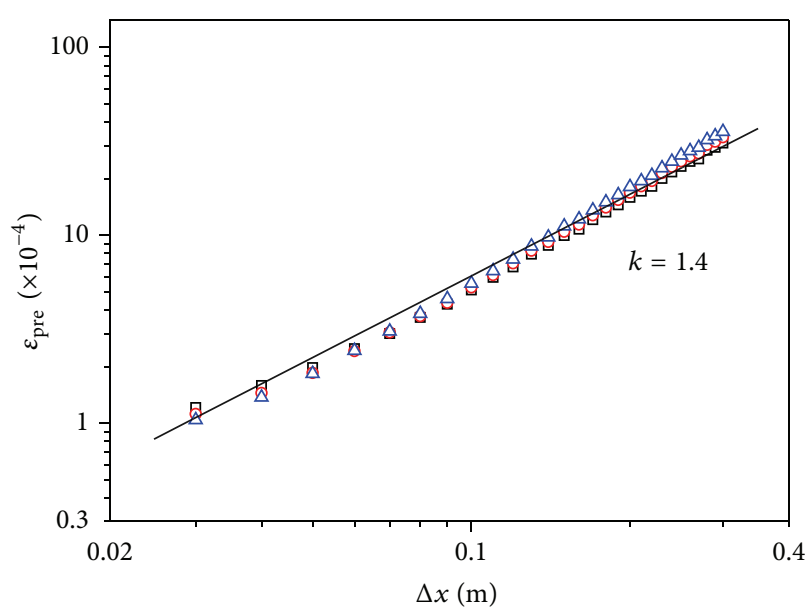

$\square$ Courant number: $0.05 \quad \Delta$ Courant number: 0.15
$\circ$ Courant number: 0.10

FIGURE 7: Sound pressure error versus particle spacing for different Courant numbers.

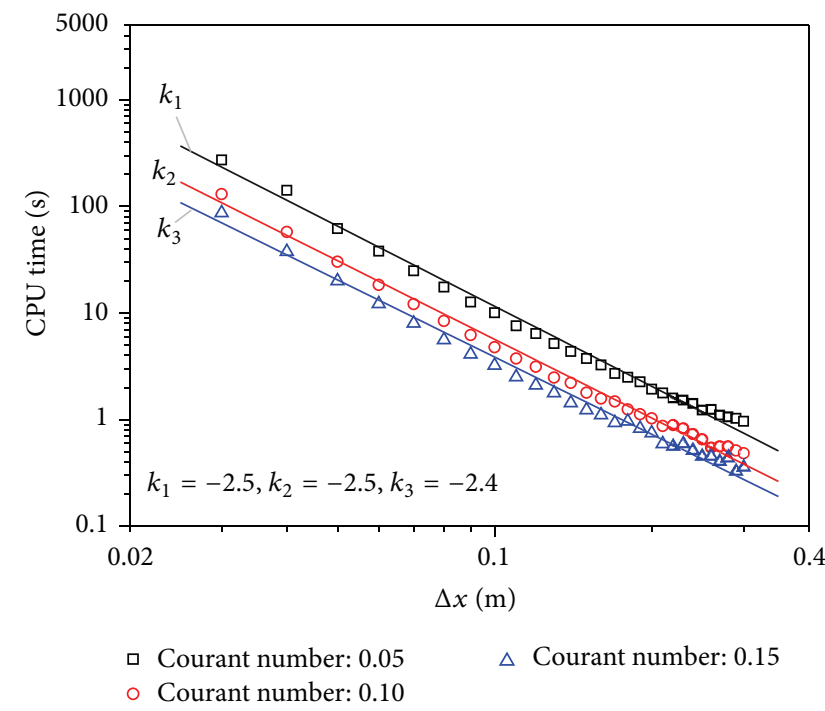

FIGURE 8: CPU time versus particle spacing for different Courant numbers.

figure also shows the linear fit lines for each Courant number, as well as the calculated slopes.

The graph shows that the CPU time logarithmically decreases from hundreds of seconds to about one second as $\Delta x$ increases from $0.03 \mathrm{~m}$ to $0.3 \mathrm{~m}$. This decreasing trend is roughly linear, with an absolute slope value of approximately 2.5. Although the CPU time is different for each Courant number, the trends are similar. Thus, an appropriate particle spacing value can be decided within a certain range by comparing the results in Figures 7 and 8 for a given application.

\section{Conclusions}

In this work, the meshfree, Lagrangian SPH method is used to solve linearized acoustic equations. The feasibility of 
the SPH method in simulating acoustic waves is evaluated using a numerical experiment of one-dimensional sound propagation model. The effect of frequency, sound pressure, and particle spacing is then subsequently discussed based on the numerical error with considering the Courant number.

(1) The results of computational cases, with the acoustic wave frequency changes from $10 \mathrm{rad} / \mathrm{s}$ to $200 \mathrm{rad} / \mathrm{s}$, show that the rising of sound frequency increases simulation error, and the increase is nonlinear.

(2) As the maximum sound pressure amplitude changes from $10 \mathrm{~Pa}$ to $200 \mathrm{~Pa}$, the rising sound pressure increases the simulation error. However, the resulting change in error is limited and marginal.

(3) Decreasing the particle spacing in the computation reduces the numerical error, while simultaneously increasing the CPU time. The trend of both changes is close to linear on a logarithmic scale. The Courant number has little effect on the error generated with different sound frequency or sound pressure, whereas the particle spacing has a more significant influence on both properties.

\section{Conflict of Interests}

The authors declare that there is no conflict of interests regarding the publication of this paper.

\section{Acknowledgments}

This study was supported by the Independent Innovation Foundation for National Defence of Huazhong University of Science and Technology (no. 01-18-140019). Y. O. Zhang is supported by the China Scholarship Council (201406160032).

\section{References}

[1] F. Ihlenburg, Finite Element Analysis of Acoustic Scattering, Springer, 1998.

[2] I. Harari, "A survey of finite element methods for timeharmonic acoustics," Computer Methods in Applied Mechanics and Engineering, vol. 195, no. 13-16, pp. 1594-1607, 2006.

[3] P. K. Kythe, An Introduction to Boundary Element Methods, CRC Press, 1995.

[4] A. Warszawski, D. Soares Jr., and W. J. Mansur, "A FEM-BEM coupling procedure to model the propagation of interacting acoustic-acoustic/acoustic-elastic waves through axisymmetric media," Computer Methods in Applied Mechanics and Engineering, vol. 197, no. 45-48, pp. 3828-3835, 2008.

[5] D. Soares Jr., "Coupled numerical methods to analyze interacting acoustic-dynamic models by multidomain decomposition techniques," Mathematical Problems in Engineering, vol. 2011, Article ID 245170, 28 pages, 2011.

[6] J. Feng, X. Zheng, H. Wang, Y. Zou, Y. Liu, and Z. Yao, "Lowfrequency acoustic-structure analysis using coupled FEM-BEM method," Mathematical Problems in Engineering, vol. 2013, Article ID 583079, 8 pages, 2013.

[7] A. Tadeu and I. Castro, "Coupling the BEM/TBEM and the MFS for the numerical simulation of wave propagation in heterogeneous fluid-solid media," Mathematical Problems in Engineering, vol. 2011, Article ID 159389, 26 pages, 2011.

[8] R. A. Uras, C. T. Chang, Y. Chen, and W. K. Liu, "Multiresolution reproducing kernel particle methods in acoustics," Journal of Computational Acoustics, vol. 5, no. 1, pp. 71-94, 1997.

[9] P. Bouillard and S. Suleau, "Element-free Galerkin solutions for Helmholtz problems: formulation and numerical assessment of the pollution effect," Computer Methods in Applied Mechanics and Engineering, vol. 162, no. 1-4, pp. 317-335, 1998.

[10] S. Wang, S. Li, Q. Huang, and K. Li, "An improved collocation meshless method based on the variable shaped radial basis function for the solution of the interior acoustic problems," Mathematical Problems in Engineering, vol. 2012, Article ID 632072, 20 pages, 2012.

[11] F. Z. Wang and K. H. Zheng, "Analysis of the boundary knot method for 3D Helmholtz-type equation," Mathematical Problems in Engineering, vol. 2014, Article ID 853252, 9 pages, 2014.

[12] L. B. Lucy, "A numerical approach to the testing of the fission hypothesis," The Astronomical Journal, vol. 82, pp. 1013-1024, 1977.

[13] R. A. Gingold and J. J. Monaghan, "Smoothed Particle Hydrodynamics-theory and application to non-spherical stars," Monthly Notices of the Royal Astronomical Society, vol. 181, pp. 375-389, 1977.

[14] F. Caleyron, A. Combescure, V. Faucher, and S. Potapov, "SPH modeling of fluid-solid interaction for dynamic failure analysis of fluid-filled thin shells," Journal of Fluids and Structures, vol. 39, pp. 126-153, 2013.

[15] P. F. Hopkins, "A general class of Lagrangian smoothed particle hydrodynamics methods and implications for fluid mixing problems," Monthly Notices of the Royal Astronomical Society, vol. 428, no. 4, pp. 2840-2856, 2013.

[16] G. W. Ma, Q. S. Wang, X. W. Yi, and X. J. Wang, "A modified SPH method for dynamic failure simulation of heterogeneous material," Mathematical Problems in Engineering, vol. 2014, Article ID 808359, 14 pages, 2014.

[17] S. Li and W. K. Liu, "Meshfree and particle methods and their applications," Applied Mechanics Reviews, vol. 55, no. 1, pp. 1-34, 2002.

[18] V. Springel, "Smoothed particle hydrodynamics in astrophysics," Annual Review of Astronomy and Astrophysics, vol. 48, pp. 391-430, 2010.

[19] M. B. Liu and G. R. Liu, "Smoothed particle hydrodynamics (SPH): an overview and recent developments," Archives of Computational Methods in Engineering. State of the Art Reviews, vol. 17, no. 1, pp. 25-76, 2010.

[20] J. J. Monaghan, "Smoothed particle hydrodynamics and its diverse applications," in Annual Review of Fluid Mechanics, vol. 44 of Annual Review of Fluid Mechanics, pp. 323-346, Annual Reviews, Palo Alto, Calif, USA, 2012.

[21] C. T. Wolfe and S. K. Semwal, Acoustic Modeling of Reverberation Using Smoothed Particle Hydrodynamics, University of Colorado, Springs, Colo, USA, 2007.

[22] P. Hahn and D. Negrut, "On the use of meshless methods in acoustic simulations," in Proceedings of the International Mechanical Engineering Congress and Exposition (IMECE '09), pp. 185-199, November 2009.

[23] Y. O. Zhang, T. Zhang, H. Ouyang, and T. Y. Li, "SPH simulation of sound propagation and interference," in Proceedings of the 5th International Conference on Computational Method (ICCM '14), Cambridge, UK, July 2014. 
[24] G. R. Liu and M. B. Liu, Smoothed Particle Hydrodynamics: a Meshfree Particle Method, World Scientific, 2003.

[25] J. J. Monaghan and J. C. Lattanzio, "A refined particle method for astrophysical problems," Astronomy and Astrophysics, vol. 149, pp. 135-143, 1985.

[26] M. Kelager, Lagrangian Fluid Dynamics Using Smoothed Particle Hydrodynamics, University of Copenhagen, Copenhagen, Denmark, 2006. 


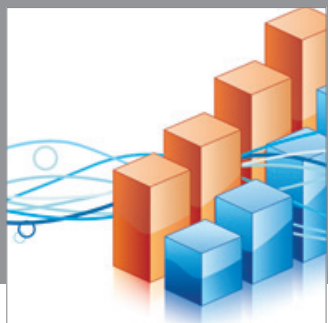

Advances in

Operations Research

mansans

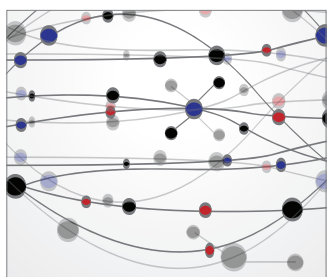

The Scientific World Journal
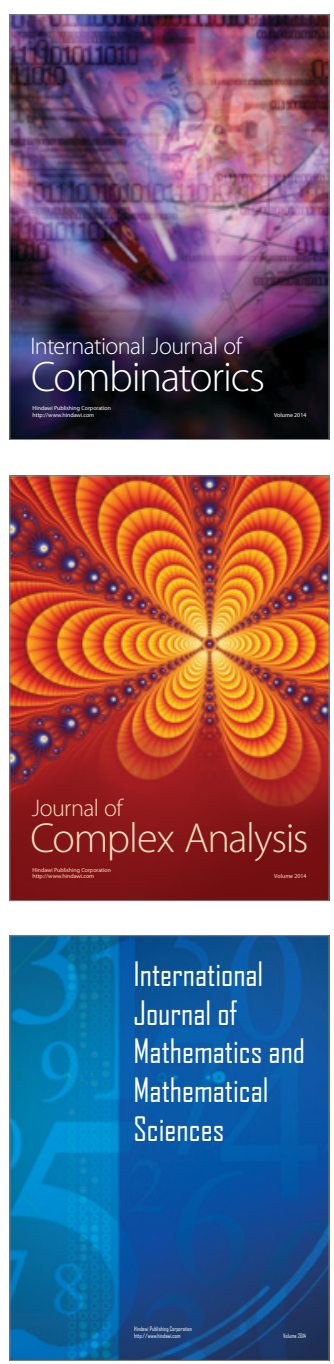
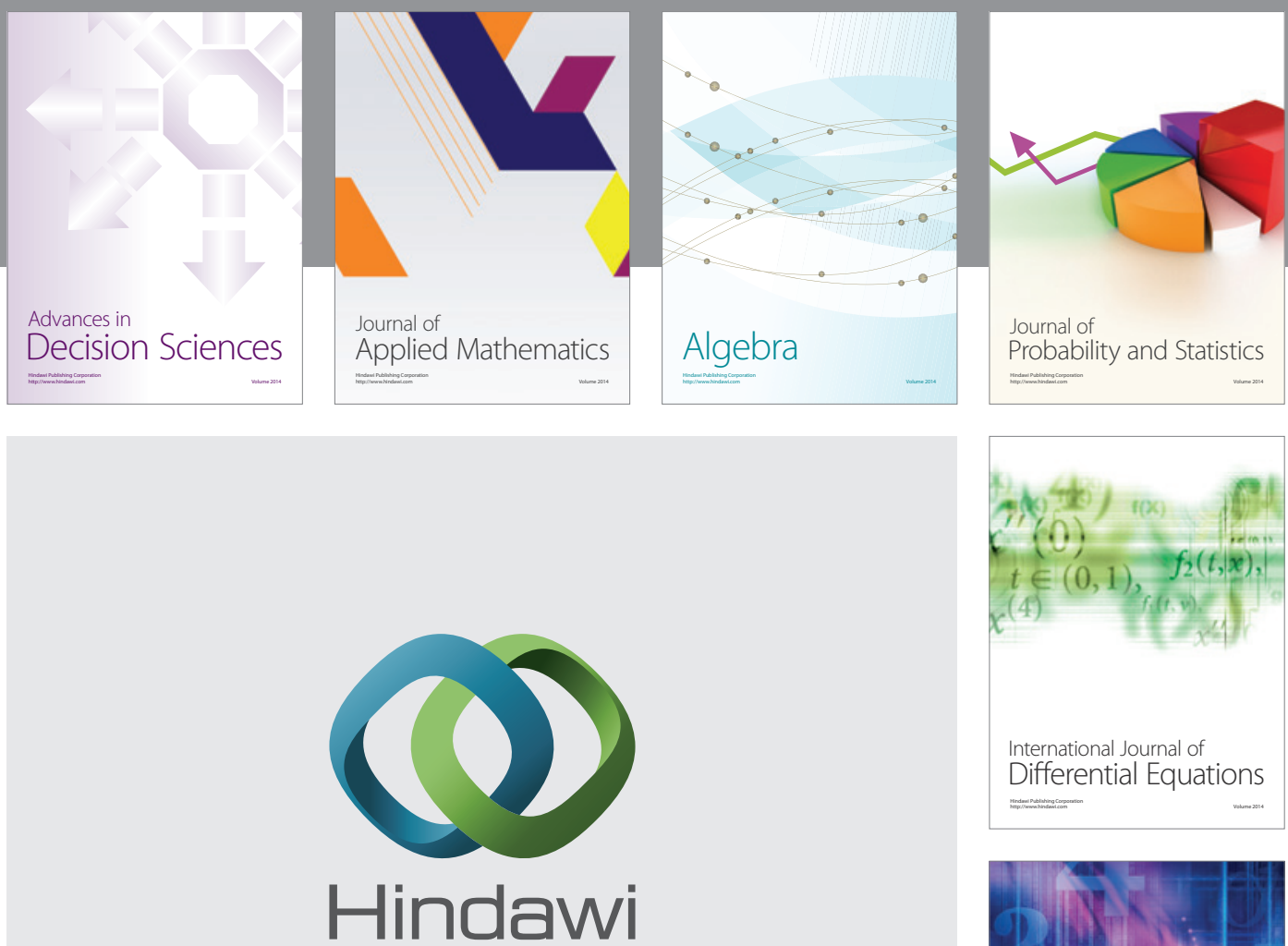

Submit your manuscripts at http://www.hindawi.com
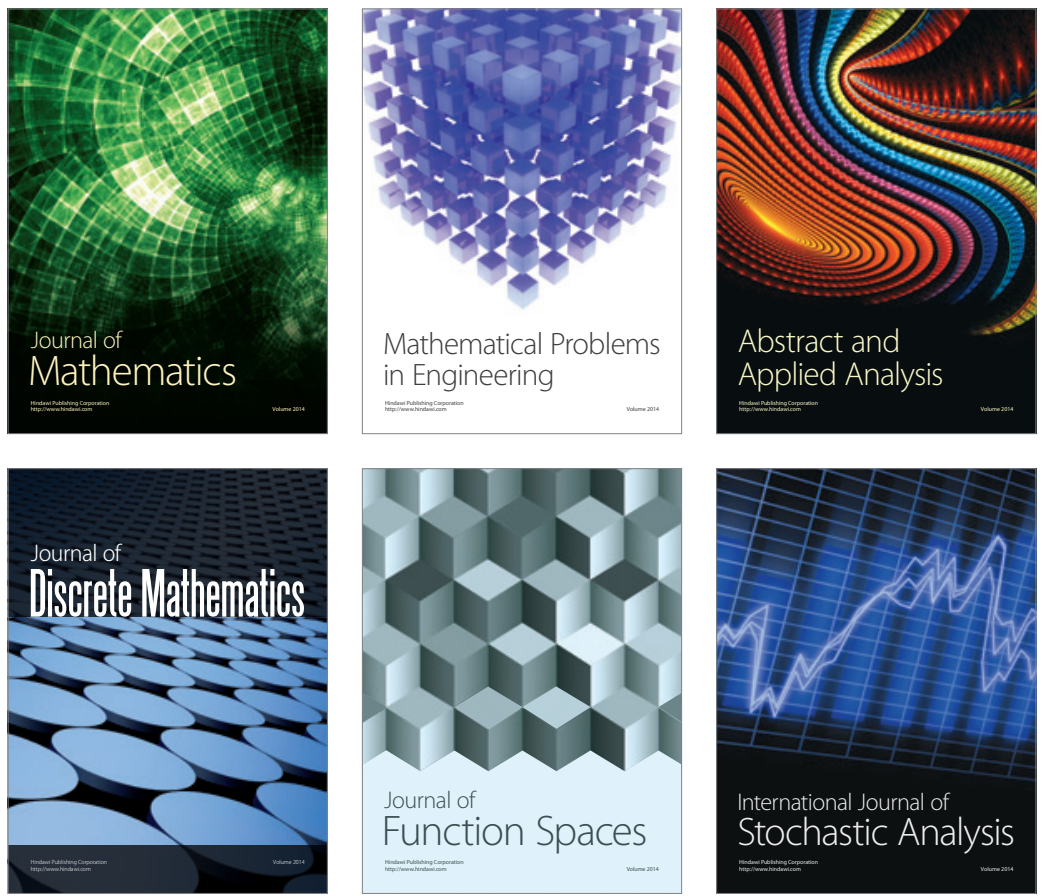

Journal of

Function Spaces

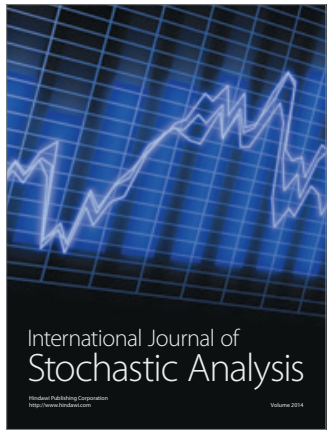

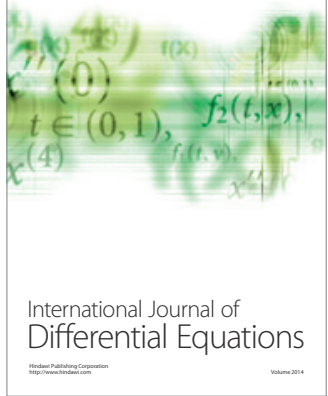
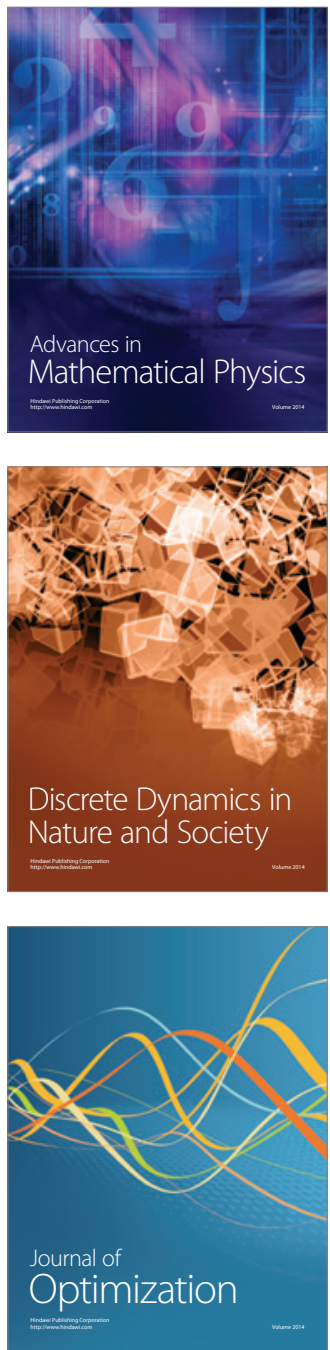